\title{
Use of Mineral Trioxide Aggregate for Non-Surgical Repair of a Furcal Perforation
}

\author{
Abdul Hakeem Qureshi ${ }^{1}$ \\ Muhammad Rameez ${ }^{2}$ \\ Haroon Rashid ${ }^{3}$
}

\begin{abstract}
:
The case report sheds light upon a non-surgical repair of a furcation defect. A 57-year old female reported for examination of tooth no. 46. The presenting complains included mild to moderate pain with small localized swelling and discharge from lingual aspect of tooth. Clinical examination revealed a discharging sinus with moderate inflammation at gingival margin on lingual aspect, with negative percussion. A diagnosis of chronic peri-radicular abscess of the tooth was made and the defect was repaired by using Mineral Trioxide Aggregate (MTA). The clinical sign and symptoms resolved after treatment within a week period.
\end{abstract}

KEYWORDS: Furcal perforation, Furcation defect, Mineral trioxide aggregate, Non-surgical repair.

HOW TO CITE: Qureshi AH, Rameez M, Rashid H. Use of Mineral Trioxide Aggregate for Non-Surgical Repair of a Furcal Perforation. J Pak Dent Assoc 2017; 26(2): 90-93.

Received: 20 January 2017, Accepted: 25 May 2017

\section{INTRODUCTION}

Perforations in Endodontics are defined as the artificial communications of the root canal system to supporting tissues of teeth or to the oral cavity [1]. Where left untreated, the perforations which are closer to the epithelial attachments, the crestal bone and those which are on the floor of pulp chamber have poor prognosis. Such perforations may causes persistent infections which may be intra-radicular or extra-radicular in nature ${ }^{1}$. The perforations, both intra and extra-radicular have been regarded as very serious complications in clinical dentistry. From a historical perspective, these perforations were one of the commonest causes of tooth extractions in the majority of cases ${ }^{2}$. These defects, which could occur due to a pathological or mechanical reasons, often require a creative approach for their management.

1. Faculty of Dentistry, Division of Conservative Dentistry, Ziauddin University, Karachi, Pakistan

2. Faculty of Dentistry, Division of Prosthodontics, Ziauddin University, Karachi, Pakistan

3. Faculty of Dentistry, Division of Prosthodontics, Ziauddin University, Karachi, Pakistan

Corresponding author: "Dr. Haroon Rashid"

<drh.rashid@hotmail.com>
A number of materials such as the dental amalgam, intermediate restorative material (IRM), zinc-oxide eugenol cement, glass-ionomer cement and mineral trioxide aggregate (MTA) have been shown to have been used for repair of such perforations ${ }^{3}$. MTA was introduced by Torabinejad et al. $^{4}$ and has been recommended as a material of choice for the repair of root canal perforations. It has been proven using different leakage approaches such as fluid filtration technique, dye-leakage model, and bacterial leakage model that the sealing ability of MTA is better as compared to many other sealing materials ${ }^{5}$. MTA has excellent anti-microbial properties, a high $\mathrm{pH}$, and excellent biocompatibility, promotes cementum growth and formation of bone, which leads to periodontal ligament regeneration around the site of injury and infection ${ }^{6}$.

The current case report sheds light upon the use of MTA for the repair of a perforation which occurred in the furcation area of a molar tooth in a private dental practice.

\section{CASE REPORT}

A 57-year old female presented at the dental practice complain of mild pain and discomfort in her tooth no. 46 with no relevant medical history. Root canal therapy had been performed on the same tooth around 2 months ago. Clinical examination revealed a discharging sinus with 
moderate inflammation at gingival margin on lingual and buccal aspects of the tooth (Figs. 1, 2). Percussion was negative while palpation on lingual aspect was slightly positive. The peri-apical radiograph revealed a mild furcal radiolucency with a root canal filling which was considered as appropriate (Fig. 3). A diagnosis of chronic peri-radicular abscess of the tooth was made due to presence of lateral perforation and a decision of perforation repair using an MTA was taken.

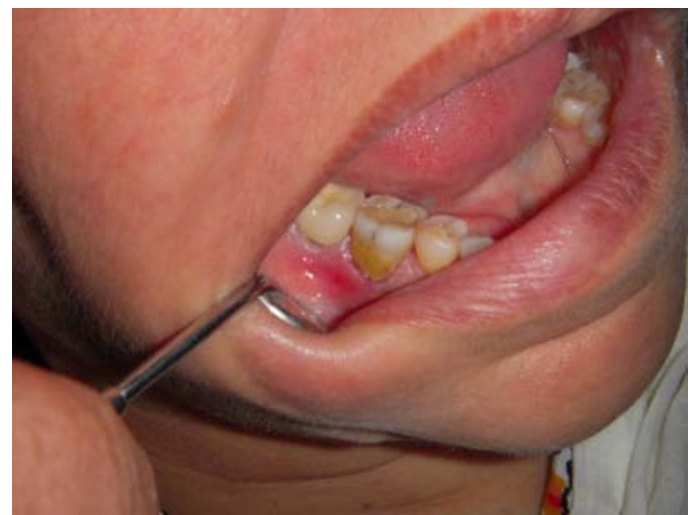

Fig. (1).

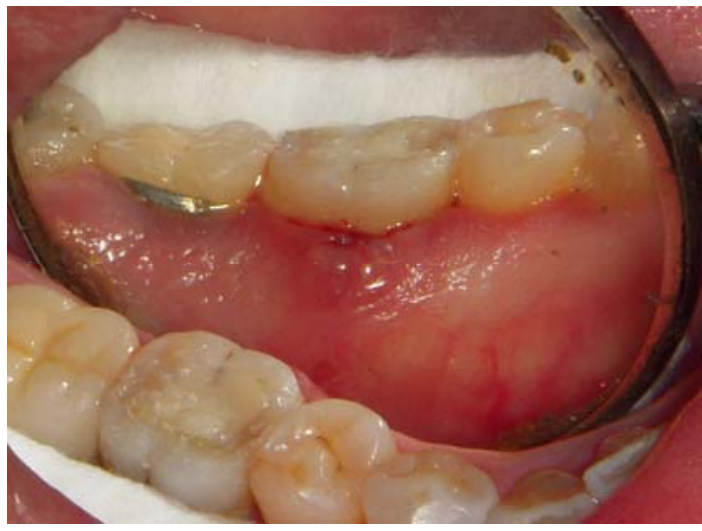

Fig. (2).

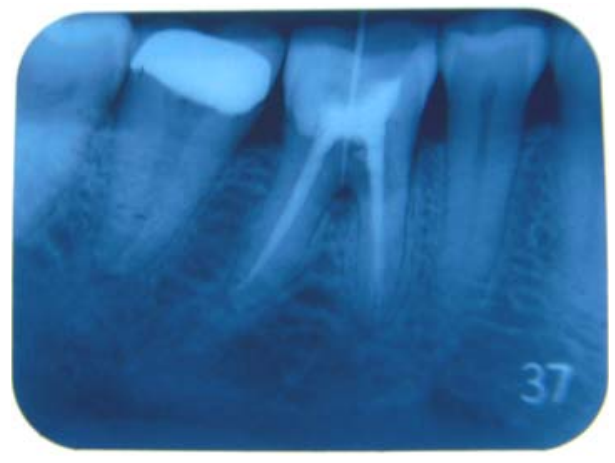

Fig. (3).

JPDA Vol. 26 No. 02 Apr-Jun 2017
Local anaesthesia was administered and rubber dam was placed to isolate the tooth. After isolation, the coronal restoration was removed by using small round-end diamond bur using a high speed air-turbine hand-piece. A round end carbide bur with a slow-speed hand-piece was used for restoration removal near the floor of the pulp chamber. After complete removal of restoration and removal of some of the gutta-percha (GP), fresh bleeding was noticed (Fig. 4). The pulp chamber was cleaned and the source of bleeding was found to be present at the lateral aspect of pulp chamber and the mesio-lingual canal of the tooth. The chamber was cleaned with 3\% Sodium Hypochlorite and the coronal 1/3 of the root end filling of the mesio-lingual (ML) canal was removed. The bleeding from the defect increased with a little more flow. The depth of the canal was measured and canal was washed and irrigated using 3\% Sodium Hypochlorite. ProRoot MTA (Dentsply, Tulsa) was mixed with distilled water to a wet sand consistency as per manufacturer's recommendations. The mixed material was placed in the canal and the pulp chamber in increments. After filling the canal completely a thin layer of MTA was also placed on the lingual wall and floor to seal the defect completely (Fig. 5). A wet cotton pellet was kept on the freshly placed MTA for 24 hours and the tooth was sealed with Cavit (3M ESPE).

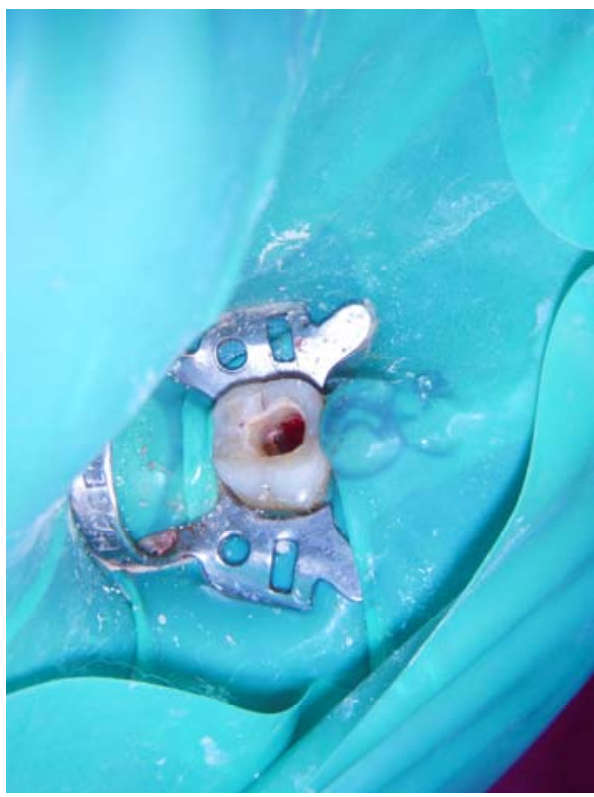

Fig. (4).

The next day, the temporary restoration was removed and a bonded resin restoration was placed. Patient was recalled after one week and then at two weeks. The treatment was considered successful as all the clinical signs and symptoms had subsided (Fig. 6). Patient has been advised to revisit the dental practice, after six months for 
further follow-up after which the definitive crown will be placed.

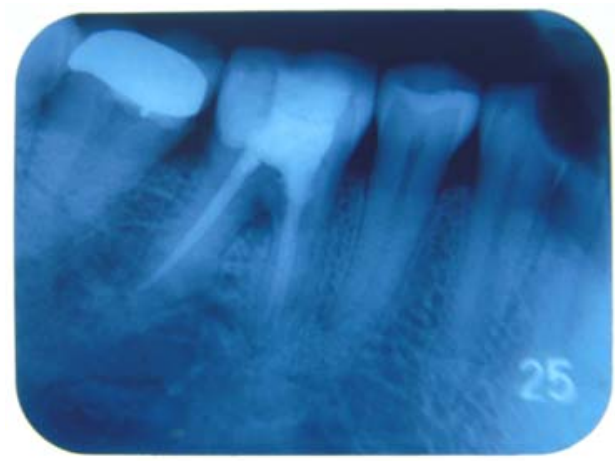

Fig. (5).

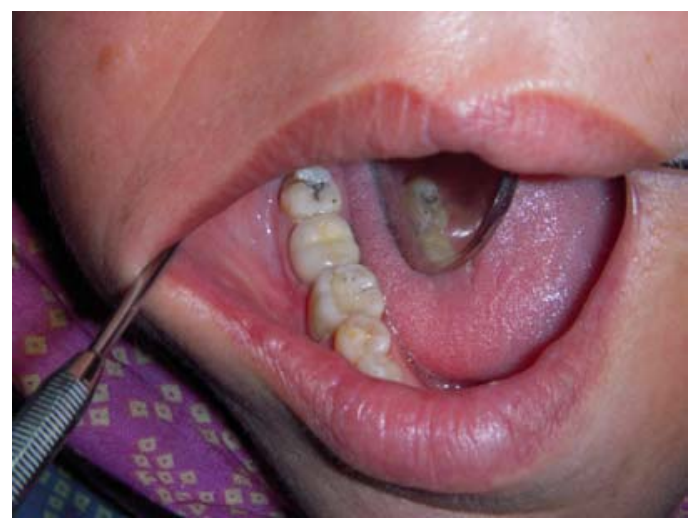

Fig. (6).

\section{DISCUSSION}

Accidental perforations of the root canal system are considered to be major complications in dentistry which may occur while performing non-surgical endodontic therapy. These complications may result in formation of granulation tissues and ultimate loss of the tooth. However, these can be managed successfully using surgical and non-surgical methods, depending upon particular characteristics of the case $^{7}$.

The prognosis is usually good if the condition is treated with a suitable material with good sealing ability and biocompatibility. Perforations can be treated with different materials and it is recommended that the material which is being used for repairs is non-toxic, non-resorbable, radioopaque, bacteriostatic or bactericidal and should provide complete seal from micro leakages ${ }^{8}$. MTA has all the required characteristics for many clinical applications i.e. as a material for obturation in tooth with open apex, for root end filling, direct pulp coverage, radicular resorption and perforation. Its suitability for managing all of these problems can be attributed to its biocompability, its low solubility, its low induction of inflammation, its capacity for creating a seal between pulpal chamber and periodontal tissues and its repair capacity ${ }^{4}$. The last of these features can be helpful due to antimicrobial properties and high pH 12.5 of MTA, which promote growth of cementum and formation of bone, which in turn allow regeneration of periodontal ligament around site of defect ${ }^{9}$.

The prognosis of lesions involving furcation also depends on the size, shape, location and period of contamination. To decrease the chances of infection, studies have revealed that best time for repair of perforation is immediately after occurrence ${ }^{10}$. The size of perforation has an important role in prognosis. Smaller the size of perforation, better will be the prognosis as smaller perforations may be easier to repair and a better seal could be achieved ${ }^{11}$. Those perforations which are closer to crestal bone and the epithelial attachment are more prone to bacterial contamination from the oral environment ${ }^{12}$. Furthermore, the apical migration of attachment may cause periodontal pockets and there may be continuous ingression of irritants from pocket, leading to subsequent failures ${ }^{13}$.

\section{REFERENCES}

1. Tsesis I, Fuss Z. Diagnosis and Treatment of accidental Root Perforations. Endodontic Topics 2006; 13: 95-107.

2. Kvinnsland I, Oswald RJ, Halse A, Gronningsaeter AG. A clinical and roentgenological study of 55 cases of root perforation. Int Endod J 1989; 22: 75-84.

3. Bains R, Bains VK, Loomba K, Verma K, Nasir A Management of pulpal floor perforation and grade II Furcation involvement using mineral trioxide aggregate and platelet rich fibrin: A clinical report. Contemp Clin Dent 2012; 3(Suppl 2): S223-S227.

4. Torabinejad M, Chivian N. Clinical applications of mineral trioxide aggregate. J Endod 1999; 25(3): 197205.

5. Parirokh $\mathrm{M}$, Torabinejad $\mathrm{M}$. Mineral trioxide aggregate: a comprehensive literature review--Part I: chemical, physical, and antibacterial properties. J Endod 2010; 36(1): 16-27.

6. Ayala S. Repair of Furcal Perforation with Mineral Trioxide Aggregate: Long- Term Follow-Up of 2 Cases. JCDA • www.cda-adc.ca/jcda • October 2008, Vol. 74, No. 8

7. Abou-Rass M, Frank AL, Glick DH. The anticurvature filing method to prepare the curved root canal. J Am Dent Assoc 1980; 101: 792-794. 
8. Sakkir N, Ahamed K, Nair MG, Joseph S, Christalin R. Management of Dilacerated and S-shaped Root Canals - An Endodontist's Challenge. J Clin Diagn Res 2014; 8(6): 22-24.

9. Savitha Adiga Ida Ataide. Non Surgical approach for Strip perforation repair using Mineral Trioxide Aggregate. J Conserv Dent 2010; 13(2): 97-101.

10. Sinai IH. Endodontic perforations: their prognosis and treatment. J Am Dent Assoc 1977; 95: 90-95.

11. Eleftheriadis GI, Lambrianidis TP. Technical quality of root canal treatment and detection of iatrogenic errors in an undergraduate dental clinic. Int Endod J 2005; 38: 725-734.

12. Gutmann JL, Harrison JW. Surgical Endodontics. Boston, MA: Blackwell Scientific Publications 1991; p. 409.

13. Fuss Z, Tsesis I, Lin S. Root resorption - diagnosis, classification and treatment choices based on stimulation factors. Dent Traumatol 2003; 19: 175182. 